\title{
Sourcing decision with capacity reservation under supply disruption risk
}

\section{Kotomichi Matsuno*}

Department of Business Design and Management, Graduate School of Creative Science and Engineering,

Waseda University, 3-4-1 Okubo, Shinjuku-ku, Tokyo, Japan

Email: sidiwu@aoni.waseda.jp

*Corresponding author

\section{Jiahua Weng}

Department of Industrial Engineering and Management,

Kanagawa University, 3-27-1 Rokkakubashi, Kanagawa-ku, Yokohama-shi, Kanagawa, Japan Email: wjh@kanagawa-u.ac.jp

\section{Xianghe Shao}

Department of Business Design and Management, Graduate School of Creative Science and Engineering, Waseda University, 3-4-1 Okubo, Shinjuku-ku, Tokyo, Japan

Email: xianghe.shao@fuji.waseda.jp

\begin{abstract}
Over the last decade, more than 50\% of organisations in the world have annually experienced a supply disruption such as geopolitical instability and transportation failure. Therefore, it has become crucial for manufacturers to comprehensively evaluate sourcing decisions not only on procurement costs, quality, and delivery but also on disruption risk. Although risk-hedging on supply disruption could be realised with evaluations on suppliers, there are no reduction effects on immense opportunity losses by manufacturers due to supply disruption. Owing to disasters such as Hurricane Katrina 2005, Great East Japan Earthquake 2011, Thailand Flood 2011, and Kumamoto Earthquake 2016, countless manufacturers have suffered from heavy losses in component procurement. In this study, as an opportunity loss mitigation countermeasure, a sourcing decision with capacity reservation (CR) is proposed for manufacturers. The effectiveness of the $\mathrm{CR}$ model is discussed by comparing it with the supplier selection and order allocation model without CR by disruption probability and supply demand relationship.
\end{abstract}

Keywords: disruption risk; sourcing decision; procurement; supply chain contract; capacity reservation.

Reference to this paper should be made as follows: Matsuno, K., Weng, J. and Shao, X. (2021) 'Sourcing decision with capacity reservation under supply disruption risk', Asian J. Management Science and Applications, Vol. 6, No. 1, pp.49-68. 
Biographical notes: Kotomichi Matsuno received his $\mathrm{MD}$ and $\mathrm{PhD}$ in Management Engineering from Waseda University, Japan, in 2012 and 2019, respectively. He is an Assistant Professor at the Department of Business Design and Management of the Graduate School of Creative Science and Engineering, Waseda University. His research interests include operations management and strategy, such as supply chain management and coordination, risk management and performance evaluation, negotiation and contract. He is a member of the Japan Industrial Management Association.

Jiahua Weng received her $\mathrm{BE}, \mathrm{ME}$ and $\mathrm{PhD}$ in Engineering from Waseda University, Japan, in 1998, 2000 and 2010, respectively. She has been a Research Associate (2003 to 2007) and Assistant Professor (2010 to 2014) of the Department of Industrial And Management Systems Engineering at Waseda University, and Associate Professor (2015-2018) in the Graduate School of Creative Science and Engineering of Waseda University. She is currently an Associate Professor of the Department of Industrial Engineering and Management at Kanagawa University. Her research interests include methods of process designing and controlling for manufacturing management systems, such as engineer-to-order production system, global sales and operation system, flexible production system and so forth. She is a member of the Japan Industrial Management Association and member of the Japan Society for Production Management.

Xianghe Shao was a master course student at the Department of Business Design and Management of the Graduate School of Creative Science and Engineering, Waseda University, Japan. He received his MD in Management Engineering from Waseda University in 2019. He currently works in Panasonic.

\section{Introduction}

Reducing procurement costs, shortening procurement lead time, and maintaining good relationships with suppliers are being considered the important matters when making decisions on supply chain construction. Currently, component procurement costs in the manufacturing industry account for about $40 \%$ to $70 \%$ of sales amount, according to Ross (2015). Building and maintaining a more competitive supply chain is considered the basis of business in the manufacturing industry. In most cases, manufacturers review and adjust their trading contracts with component suppliers for the next year annually, based on their long-term goals and annual procurement funding. In the past, during the sourcing decision-making by manufacturers, suppliers, variety, and quantity of components from each supplier were decided with an integrated evaluation of procurement cost, quality, and delivery time. As a result, the minimum number of suppliers at the lowest procurement costs was decided as the procurement source. However, as shown in Figure 1, there have been many cases of disasters in the recent years, such as the Hurricane Katrina 2005, Great East Japan Earthquake 2011, Thailand Flood 2011, Kumamoto Earthquake 2016 that have interrupted procurement activities and resulted in a shortage of productions components planned by manufacturers. Ho et al. (2015) presented that supply disruptions cause significant losses to countless manufacturers, making it difficult for them to survive in the product market. Sourcing decision-making 
with consideration of disruption risk is becoming increasingly important for manufacturers.

Figure 1 Supply chain disruptions due to disasters (see online version for colours)

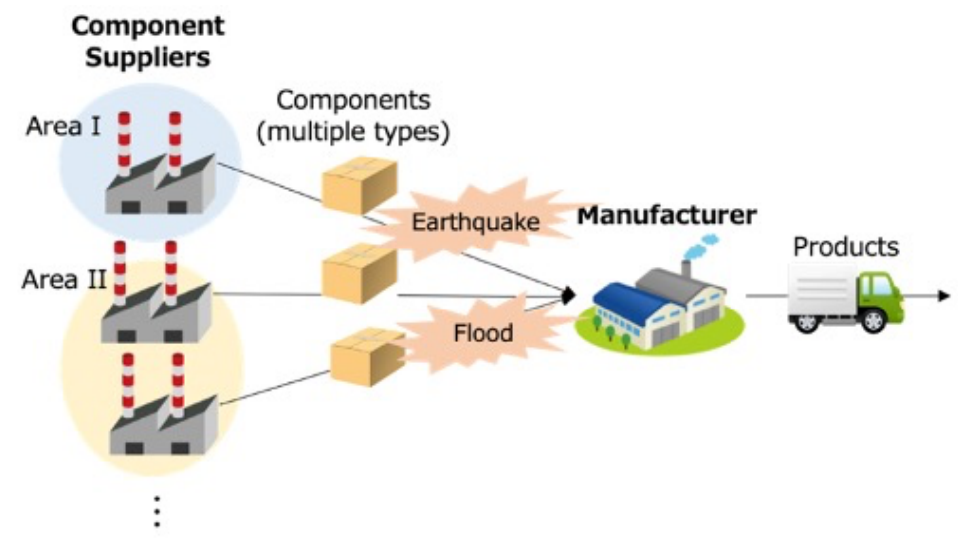

Research on supply chain disruption risk management (SCDRM) to deal with supply chain disruptions has been attracting attention and the number of studies has been increasing rapidly. However, research in the field of SCDRM is still in its infancy. Three main countermeasures that have been discussed for SCDRM are sourcing decision with assessing the risk of disruption, preparing response plan in advance and taking quick action after the fact. To build a supply chain that is resilient to disruption risk, the first two of the three countermeasures are particularly important, but there are also many challenges. Many articles such as Torabi et al. (2015) and Hamdi et al. (2016) focused on supplier evaluation and selection by considering not only procurement cost, quality, and delivery time, but also the disruption risks. However, although risk-hedging on supply disruption could be realised with such evaluations on suppliers during procurement planning, there are no mitigation effects on immense opportunity losses for manufacturers due to component procurement disruptions. Adjusting inventory levels and increasing number of suppliers for placing orders are considered as the response plans in advance. Tomlin (2006) and Schmitt et al. (2010) focused on inventory levels adjustment for disruption risk-hedging. Dadaet et al. (2007) and Chopra et al. (2007) discussed the loss mitigation effect with multiple sourcing under disruption risks environment. The biggest challenge in preparing response plan in advance is how to minimise the impact on normal operations and minimise the cost of countermeasures in the usual time without disruptions.

In this study, to realise opportunity loss mitigation for manufacturers under supply disruption due to disasters, a sourcing and procurement plan is proposed based on supply capacity reservation (CR). When concluding components procurement contracts with each supplier, besides transaction volume and transaction price, manufacturer could pay CR fee to reserve the production capacity of each supplier in advance up to a certain upper limit to obtain more components than the transaction volume if necessary. Furthermore, the CR can help both manufacturer and supplier to realise a win-win relationship. For manufacturers, immense opportunity losses due to component procurement disruptions could be mitigated. For suppliers, CR fee could be achieved from the manufacturer as the additional income in the usual time. Comparing with the 
response plans in advance such as adjusting inventory levels and increasing number of suppliers for placing orders, less impact on normal operations and less cost for countermeasures in the usual time can be realised by procurement plan based on CR.

We clarify the effectiveness and applicable conditions of the proposed plan after calculating the optimal number of suppliers and the optimal component order allocation. The remainder of this paper is organised as follows. Section 2 provides a review of the literature. Section 3 presents the basic model of sourcing decision. Section 4 presents the CR model. The algorithm is discussed in Section 5. Section 6 presents the experimental summary and results of numerical experiment. The paper is concluded in Section 7.

\section{Literature review}

In this section, relevant previous studies on SCDRM, in particular supply chain design and construction with considering disruption risk are introduced. Problems and challenges of the previous studies as well as the proposal of CR contract are discussed to show the positioning of this study.

Supplier selection and allocation is one of the most important decisions in component procurement. It directly impacts the total supply chain performance as well as the management activities of manufacturers. Many previous studies have discussed the decision-making of supplier selection and allocation with an overall evaluation, including supply disruption risks. Amir et al. (2018) discussed supplier selection from all candidate suppliers to place orders by comprehensive scale assessment, including various risks and component procurement costs. Masuda and Mizuno (2017) built several supply chain structural models and discussed the balance between supply chain efficiency and robustness under supply disruption risks. Moghaddam (2015) treated the components procurement problem as a recovery purchasing problem of reused components under the uncertainties of demand, supply capacity, and volumes of recovered components accounted for. The problem was dealt with as a multi-objective function optimisation problem with randomly varying weights of the objective functions of cost, quality, delivery, and economic risks. Abginehchi and Farahani (2010) discussed the problem of optimal supplier number by considering the component inventory level when placing an order under procurement lead-time uncertainty. Meena et al. (2011) proposed a supplier selection method with the minimum total costs, including opportunity loss, under a risk scenario. It is based on the assumption that the component procurement price of each supplier is the same, and the component order volume from each supplier is also the same. Chopra et al. (2007) focused on the decoupling recurrent supply risk and disruption risk when planning appropriate mitigation strategies. It is concluded that firms should order more from a reliable source and less from a cheaper but less reliable source if most of the supply risk growth comes from an increase in disruption probability.

Supplier selection and component allocation with supply risk evaluation are important in sourcing decision-making. However, if one or several selected suppliers are disrupted in practice, even though the sourcing plan is decided by a comprehensive and accurate assessment, the components cannot be obtained by manufacturers, resulting in a huge opportunity loss. As introduced in Section 1, preparing response plan in advance which is the second countermeasure for SCDRM is necessary. As loss mitigation approaches, supplier diversification strategy and inventory level adjustment have been discussed in some previous studies. Meena and Sarmah (2016) conducted their study under the 
assumption that the manufacturer could get replenished from other suppliers when supply disruption occurs. They determined the number of suppliers and component volume allocated to each supplier by the object function of minimum total cost and by considering the supply disruption probability of each supplier that was selected based on a risk scenario. However, it is rather difficult to obtain enough components from other suppliers when supply disruption occurs without any promise beforehand. Tomlin (2006) studied a single-product setting in which a firm can source from two suppliers, one of the suppliers is unreliable and the other is reliable. It is proved that the firm would pursue mitigation by carrying inventory, mitigation by single sourcing from the reliable but more expensive supplier as disruption management strategy in the case that the reliable supplier has no flexibility on volume and the unreliable supplier has infinite capacity. Schmitt (2011) focused on a multi-echelon supply chain. Inventory holding and site diversification are considered countermeasures to mitigate delivery delays when disruption occurs. The results are discussed based on a stochastic model. The problem is that manufacturers should bear a significant burden, even if no procurement disruption occurs.

Many previous studies have dealt with sourcing decisions and component allocation problems, but few considered countermeasures in the event of a supply disruption. As discussed above, few studies that have focused on countermeasures lack a sense of reality. As a loss mitigation approach for production investment and component procurement under uncertainties, supply chain coordination with CR contract has been discussed in many articles like Jin and Wu (2007), Cheaitou and Cheaytou (2019), Takemoto and Arizono (2019) and so on. In most of the previous articles, CR contract with decision variables including capacity volume, reservation volume and reservation fee was proposed to a supplier and a buyer under non-cooperative relationship. Each decision variable is determined separately based on individual profit functions. Effectiveness of CR contract has been confirmed in such non-cooperative supply chains under uncertainties in industries such as apparel and high-tech industries. Hou et al. (2017) focused on a supply chain including a manufacturer who faces stochastic demand, an overseas module supplier and two local module suppliers. The overseas supplier offers quality products while being susceptible to disruption risks. On the contrary, if the local suppliers with high reliability offer products that are of inferior quality, it may result in lower market acceptance. Optimal order volume and reservation volume of manufacturer based on CR contract have been derived. Moreover, merits of backup sourcing with CR for managing demand uncertainties and supply disruption risks are discussed. Effectiveness of CR contract was confirmed in such supply chain situation, however, sourcing decision including optimal number of suppliers and supplier selection for building supply chain has not been discussed. Moreover, manufacturers should decide not only the procurement volume, but also the item types in general, but it has not been fully considered in relevant studies.

In this study, after modelling the sourcing decision-making under multiple candidate suppliers and multiple types of components situation, a new sourcing decision model based on $\mathrm{CR}$ is proposed. Countermeasure of preparing response plan in advance and sourcing decision with assessing the risk of disruption for SCDRM can be realised with the proposed model. Effectiveness of proposed model is discussed by manufacturer's opportunity loss reducing with supply disruption risk in different levels under a onemanufacturer multiple supply source structure. 


\section{Sourcing decision-making model}

In high-tech industry such as smart phone or tablet PC, manufacturer procures core components from several main suppliers. Next-generation components with higher performance are being introduced to the component market one after another, at the same time, component supply environment is changing dramatically due to intense competition among component suppliers. As a result, it is necessary for product manufacturer to make sourcing decision to reconstruct supply chain frequently. In most cases, manufacturer review and adjust his trading contracts with component suppliers for the next year annually.

In this section, after summarising the assumptions of this study, sourcing decision-making model for the manufacturer is formulated.

\subsection{Prerequisites}

The one-manufacturer multiple suppliers supply chain structure is considered in this study. As discussed before, procurement planning and re-contract including sourcing decision is held once a year. The types and volumes of the components required are decided by manufacturer according to the production plan of products. Manufacturer evaluates all candidate component suppliers to make sourcing decision for next year. All candidate component suppliers whose attributes such as component quality and delivery could meet the requirements of the manufacturer, but they have different features on procurement costs per unit of each component, disruption probabilities, fixed management costs, and procurable volumes.

1 One-manufacturer multiple suppliers supply chain structure.

2 The types and volumes of the components required are decided by the manufacturer.

3 Multiple candidate component suppliers were considered in the model. The attributes of all candidate suppliers, such as component quality and delivery, could meet the requirements of the manufacturer.

4 All candidate suppliers have different features on procurement costs per unit of each component, disruption probabilities, fixed management costs, and procurable volumes.

5 Manufacturers review and adjust their trading contracts with component suppliers for the next year annually. So, procurement planning including sourcing decision is held once a year. Trading contract between the manufacturer and supplier can be considered as a one-year contract. Both the span and cycle of the contract are 1 year.

\subsection{Objective functions and constraints}

\section{Notations}

$s \quad$ Supplier ID $(s=1,2,3, \ldots, S)$

$i \quad$ ID of component types $(i=1,2,3, \ldots, I)$

$D_{i} \quad$ Demand of component $i$ 
$M_{S} \quad$ Fixed supplier management cost $s$

$P_{i s} \quad$ Price of component $i$ from supplier $s$ (per unit)

$Q_{i s}^{\min } \quad$ Minimum procurement volume of components $i$ from supplier $s$

$Q_{i s}^{\max } \quad$ Maximum procurement volume of components $i$ from supplier $s$

$L_{i} \quad$ Opportunity loss of components $i$ (per unit)

Trans $_{i s}$ Transportation cost of components $i$ from supplier $s$ (per unit)

$\theta_{s} \quad$ Disruption probability of supplier $s(\%)$

$a \quad$ ID of interrupted suppliers among selected suppliers $(a=1,2,3, \ldots, A)$

$b \quad$ ID of uninterrupted suppliers among selected suppliers $(b=1,2,3, \ldots, B)$

$A_{a} \quad$ The interrupted supplier group (ex. $A_{1}$ indicates the group of any one interrupted supplier)

$B_{b} \quad$ The uninterrupted supplier group (ex. $B_{1}$ indicates the group of uninterrupted suppliers when any supplier is interrupted).

\section{Decision variable}

$n \quad$ Number of selected suppliers

$Z X_{s}$ If components are sourced from the supplier $s, Z X_{s}=1$, otherwise $Z X_{s}=0$

$Q_{i s} \quad$ Procurement volumes of components $i$ from supplier $s$.

\section{Objective functions}

The objective function, which is the minimum total procurement cost including the opportunity cost, is shown below:

$$
T P C_{\min }=\text { Fixed mgmt. cost }+ \text { pur. cost }+ \text { trans. cost }+ \text { oppty. loss }
$$

Fixed management cost, as shown in formula (2), is the fixed cost involved in the process of managing supplier activities and contracts. The purchasing cost is the total cost of purchasing components from all suppliers. As shown in formula (3), it can be calculated by multiplying the unit price of each variety by the component volume to be purchased. Purchasing cost here refers to a normal situation without considering supply disruption. Transportation costs can be calculated by multiplying the unit transportation cost of each component from one supplier to the manufacturer by the quantity of components purchased from the supplier.

$$
\begin{aligned}
& \text { Fixed management. cost }=\sum_{s=1}^{S} \sum_{i=1}^{I} Z X_{s} \times M_{s} \\
& \text { Purchasing cost }=\sum_{s=1}^{S} \sum_{i=1}^{I} P_{i s} \times Q_{i s}
\end{aligned}
$$




$$
\text { Transportation cost }=\sum_{s=1}^{S} \sum_{i=1}^{I} \operatorname{Trans}_{i s} \times Q_{i s}
$$

The opportunity loss is the loss that occurs when the manufacturer is unable to procure products because of supply disruption. The volume of components that cannot be supplied at the time of supply disruption can be calculated using the following formula (5), which are the multiplication of the expected insufficient volume and the disruption probability of one supplier. The opportunity cost of the case in which any one supplier gets disrupted could be calculated by formula (6). Similarly, the opportunity cost of the case in which any two suppliers get disrupted could be calculated by formula (7). As in the formulas above, the opportunity cost when any $n$ suppliers are interrupted can be calculated with formula (8). Therefore, the opportunity loss can be expressed with formula (9), which is the sum of $C 1, C 2, \ldots, C n$.

$$
\begin{aligned}
& \sum_{i=1}^{I}\left[D_{i}-\min \left(D_{i}, \sum_{b=1}^{B} Q_{i b}^{\max }\right)\right] \times\left[\prod_{a \in A_{1}} \theta_{a} \times \prod_{b \in B_{1}}\left(1-\theta_{b}\right)\right] \\
& C 1=\sum_{i=1}^{I} L_{i} \times \sum\left\{\left[D_{i}-\min \left(D_{i}, \sum_{b=1}^{B} Q_{i b}^{\max }\right)\right] \times\left[\prod_{a \in A_{1}} \theta_{a} \times \prod_{b \in B_{1}}\left(1-\theta_{b}\right)\right]\right\} \\
& C 2=\sum_{i=1}^{I} L_{i} \times \sum\left\{\left[D_{i}-\min \left(D_{i}, \sum_{b=1}^{B} Q_{i b}^{\max }\right)\right] \times\left[\prod_{a \in A_{2}} \theta_{a} \times \prod_{b \in B_{2}}\left(1-\theta_{b}\right)\right]\right\} \\
& C 3=\sum_{i=1}^{I} L_{i} \times \sum\left\{\left[D_{i}-\min \left(D_{i}, \sum_{b=1}^{B} Q_{i b}^{\max }\right)\right] \times \prod_{a \in A n} \theta_{a}\right\}
\end{aligned}
$$

\section{Constraints}

1 Demand constraints of the components

The total volumes of component $i$ that could be procured from all selected suppliers are equal to the demand of the component $i$.

$$
\sum_{s=1}^{S} Q_{i s}=D_{i} \quad \forall i
$$

\section{Procurement constraints}

The volume of components procured from supplier $s$ should not be less than the minimum volume, and no more than the maximum quantity decided by the supplier $s$.

$$
Q_{i s}^{\min } \leq Q_{i s} \leq Q_{i s}^{\max } \quad \forall i, s
$$


3 Constraints on the number of suppliers

The number of suppliers decided should not exceed the total number of candidate suppliers.

$$
n \leq S
$$

\section{Production CR}

As discussed in Section 2, CR could be considered as a loss mitigation countermeasure for supply disruption. With the reserved supply capacity, the manufacturer could obtain the expected volume of component from the suppliers who are not in a disruption situation at the time of one or more supply disruptions. On the other hand, with CR, in addition to the planned procurement volume of component, the manufacturer and supplier should reach an agreement on more production capacity for the contract duration. Therefore, an extra reservation fee should be added to procurement costs.

Figure 2 shows the price of procurement volume and the price of the reservable capacity of the component $i$ from the supplier $s$. With the reserved capacity from the supplier $s$, the manufacturer could purchase more components than $Q_{i s}^{\max }$ to reduce opportunity loss at the time of other supply disruptions. In general, a contract fee should be paid by the manufacturer to reserve the production capacity of suppliers at the time of contract connection.

\section{Notations}

$C F_{i s} \quad$ CR contract fee of components $i$ from supplier $s$

$K_{i s} \quad$ Capacity utilisation cost (per unit) of components $i$ from supplier $s$

$E Q_{i s} \quad$ Upper limit of CR of the supplier $s$

$R E S_{i s} \quad$ CR cost of components $i$ from supplier $s$

$C_{i s} \quad$ Upper limit of procurement volume of component $i$ from supplier $s$

$$
\begin{aligned}
& \operatorname{Res}_{i s}=K_{i s} \times E Q_{i s} \\
& C_{i s}=Q_{i s}^{\max }+E Q_{i s}
\end{aligned}
$$

Since the probability of multiple suppliers being disrupted at the same time among the candidate suppliers is extremely low, the case when one of the suppliers is disrupted must be discussed as a high priority.

$E Q_{i s}$ is decided by the manufacturer by considering the case that if one supplier is disrupted, the components supplied by the supplier could be averagely covered by other candidate suppliers. For example, suppliers 1, 2, and 3 are decided as procurement sources, and the reservation capacity of supplier $1\left(E Q_{i 1}\right)$ can be determined as follows: If either supplier 2 or supplier 3 is disrupted, as the total volume of the component $i$ should be supplied by supplier 2 and supplier 3 is $\left(Q_{i 2}+Q_{i 3}\right)$, the average of $\left(Q_{i 2}+Q_{i 3}\right)$ should be shared between the remaining two undisrupted suppliers. Therefore, the reservation capacity of supplier $1\left(E Q_{i 1}\right)$ can be determined by formula (15). Similarly, when $N$ 
suppliers $1,2, \ldots N$ are selected as the supply sources, the reservation capacity of supplier $1\left(E Q_{i 1}\right)$ can be expressed by formula (16). Capacity utilisation cost should be considered as fixed management costs by using CR. Therefore, formula (2) should be changed to formula (17) with CR.

$$
\begin{aligned}
& E Q_{i 1}=\left(Q_{i 2}+Q_{i 3}\right) / 4 \\
& E Q_{i 1}=\left(Q_{i 2}+Q_{i 3}+\ldots Q_{i N}\right) /(n-1)^{2} \\
& \text { Fixed management cost }=\sum_{s=1}^{S} \sum_{i=1}^{I} Z X_{s} \times\left(M_{s}+E Q_{i s} \times K_{i s}\right)
\end{aligned}
$$

Figure 2 Relation between component volume/capacity and price (per unit) (see online version for colours)

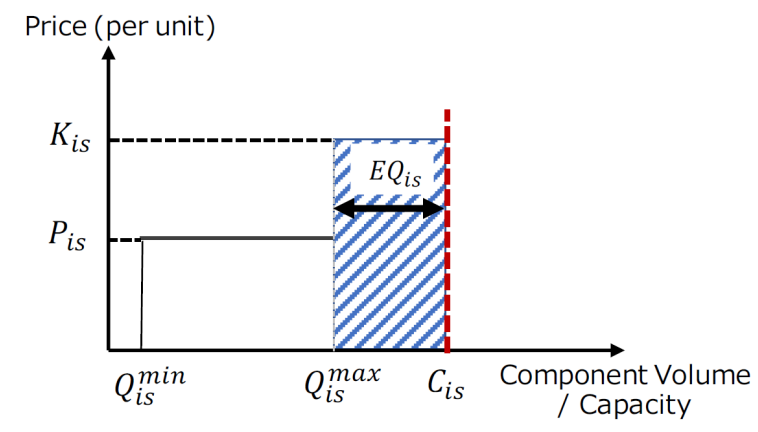

\section{A tabu-search-based algorithm for decision-making}

Prohibitions are introduced to discourage the search from coming back to previous-visited solutions. Tabu-search (TS) is expected to search more neighbourhood solutions with fewer search times. In general, procurement policy and plan for the next year is determined during a procurement strategy meeting. So, it is necessary to create and re-create appropriate and reliable procurement plans in a short time while evaluating candidate component suppliers in the meeting.

In this study, the decision-making algorithm is designed based on TS with considering the search efficiency.

\subsection{Initial solution creation}

There are two steps to creating an initial solution. First, we create an initial supplier combination randomly with the determined number of suppliers. Second, the procurement amount of each component from each supplier is created at random. Then, we adjust the procurement amount of each component from each supplier randomly while considering whether the demand of components could meet the minimum and maximum procurable volume of each component of each supplier. 


\subsection{Solution improvement}

\subsubsection{Conditions for supplier combination adjustment}

The conditions for adjusting the combination of suppliers are decided based on whether the volume of components allocated could meet with the maximum and minimum procurable volume of each supplier.

There are also two steps before supplier combination adjustment. First, we evaluate the unit price of components of each supplier. For example, 3 suppliers from the candidate suppliers are selected as the initial combination. The supplier with the lowest component price should be ranked first, and the supplier with the highest component price should be ranked third. In the case of three component types, there are three evaluation results. Second, we confirm the supplier combination adjustment conditions based on the evaluation results. Table 1 shows the cases that should be considered to adjust supplier combination.

Table 1 Cases of supplier combination adjustment conditions

\begin{tabular}{lccc}
\hline \multirow{3}{*}{ Cases } & \multicolumn{3}{c}{ Valuation rank of suppliers } \\
\cline { 2 - 4 } & 1st (lowest price) & 2nd (medium price) & 3rd (highest price) \\
\hline 1 & Upper limit & Upper limit & \\
2 & & Lower limit & Lower limit \\
3 & Upper limit & & Lower limit \\
\hline
\end{tabular}

The three cases in Table 1 can be considered as the combination adjustment conditions. The upper and lower limits in Table 1 are procurement restrictions for each supplier. In case 1, the allocated volume of components is equal to the upper limit procurement amount of supplier 1 with the highest valuation as well as supplier 2 with the medium price. Because it is not possible to adjust the volume of the component to a supplier with a lower unit price, the supplier combination is considered to be adjusted. In case 2, the allocated volume of components is equal to the lower limit procurement amount of supplier 3 with the lowest price as well as supplier 2 with the medium price. In addition, it is not possible to adjust the volume of components to a supplier with lower unit prices, the supplier combination should be adjusted. Case 3 shows the same situation wherein it is not possible to adjust the volume of the component to a supplier with lower unit prices, and therefore the supplier combination should be adjusted.

\subsubsection{Supplier combination adjustment algorithm}

For the initial supplier combination, the average procurement per unit cost of all components is calculated and the supplier with the highest cost should be selected as the candidate for improvement. To substitute the candidate supplier for the improvement search one by one from unselected suppliers. Next, we check whether component demand could meet the constraints on minimum and maximum procurement volumes of each new supplier combination and also check whether they are on the tabu list. If the constraints can be met and the supplier combinations do not exist in the tabu list at the same time, they are assumed to be the neighbourhood solutions and the total procurement cost of each supplier combination is to be calculated. After the calculation, we compare the results and add the supplier combination with the lowest average unit cost to the tabu list. 
If the conditions for updating supplier combination are not met, this implies that there is room for reducing procurement costs by adjusting the procurement volume from the suppliers; the allocation of each component will be adjusted based on the minimum adjustment amount. In this study, based on the aforementioned supplier evaluation, five units of components as the minimum adjustment amount would be moved from the supplier with the highest component unit price to suppliers with lower component unit price within the range of maximum and minimum procurement of each type of component.

Figure 3 Process of supplier combination improvement (see online version for colours)

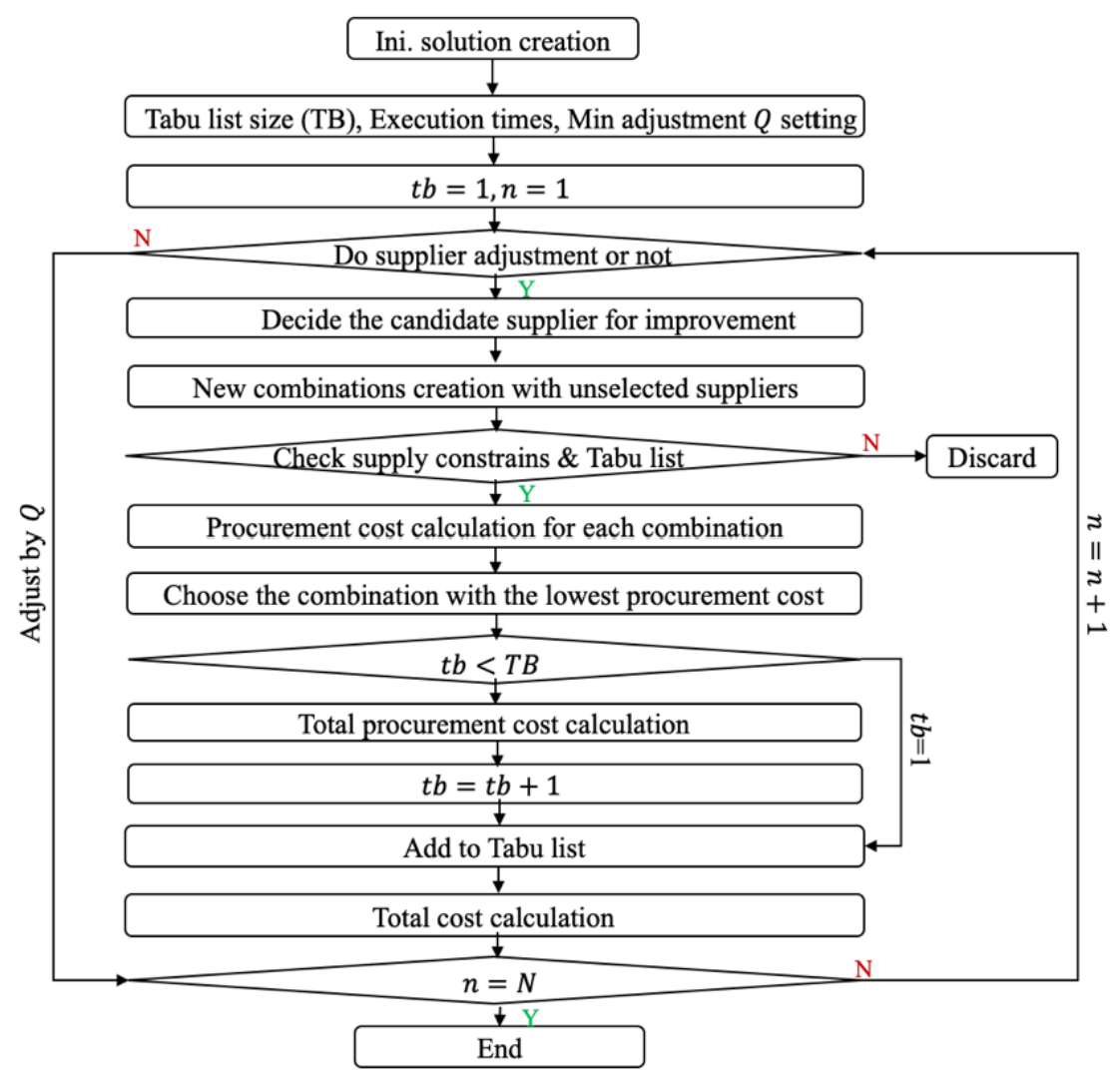

The details of the improvement process are shown in Figure 3. There are five main steps in this process.

Step 1 Check the initial supplier combination and confirm whether the condition is necessary to update the supplier combination or not. If so, proceed to step 2 , otherwise, proceed to step 4.

Step 2 Calculate the average procurement per unit cost of all components and decide on the candidate with the highest cost for improvement. Create new supplier combinations by selecting the unselected suppliers one by one to replace the candidate supplier. Confirm the combinations of whether component demand could meet the constraints on minimum and maximum procurement volumes of 
each supplier and also check whether the combinations are on the tabu list or not. If the constraints of procurement volume can be met and the supplier combinations do not exist in the tabu list at the same time, replace the candidate supplier and proceed to step 3. Otherwise, take no action. Figure 4 shows an example of neighbourhood solution creation.

Step 3 Calculate the average procurement unit cost of all components of the new supplier combinations. Add the new supplier combination with the lowest unit cost to the tabu list and update the tabu list. Figure 5 shows an example of the tabu list update.

Step 4 Evaluate the procurement cost of each component of each supplier. Adjust the component volume from the supplier with the highest component procurement cost to the supplier with the lowest component procurement cost based on the minimum adjustment amount.

Step 5 Update the execution times of the search. If the execution time $n$ is less than the total search time $N$, proceed to step 2. Otherwise, end the execution of the search.

Figure 4 An example of neighbourhood solutions creation (see online version for colours)

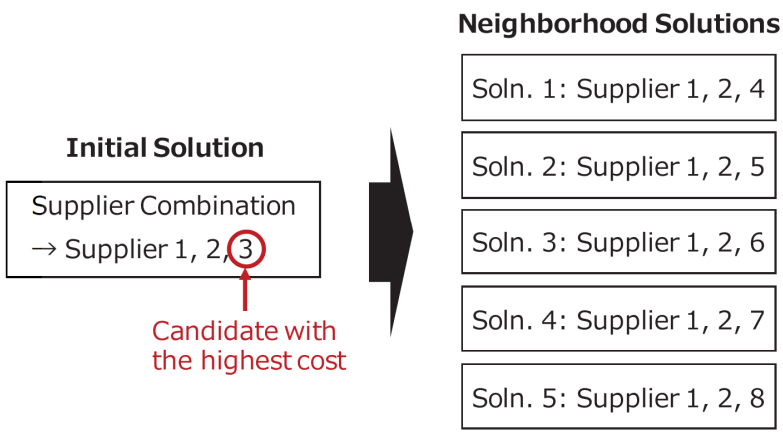

Figure 5 An example of tabu list update (see online version for colours)

Tabu List (Before)

No. 1: Supplier 2, 3, 4

No. 2: Supplier 2, 3, 5

No. 3: Supplier 2, 3, 6

No. 4: Supplier 3, 4, 7

No. 5: Supplier 1, 3, 8
Tabu List (After)

No. 1: Supplier $1,2,6$ The best nbhd.

No. 2: Supplier 2, 3, 5

No. 3: Supplier 2, 3, 6

No. 4: Supplier 3, 4, 7

No. 5: Supplier 1, 3, 8 


\subsection{Performance verification}

The performance of the proposed algorithm in Section 5.2 is verified and the experimental results are discussed in this sub-section.

In general, if the location of a supplier or the transportation route from a supplier is unstable, the component price of the supplier is considered to be slightly cheaper among the candidate suppliers. The data for the experiment created considering such scenarios are shown below.

\section{Detail of experiment data}

- $\quad$ Number of candidate suppliers: 8

- Type of components: 3

- Demand for each component type: 400, 300, 200

- $\quad$ Range of fixed management cost: 1,500-2,500

- Range of minimum procurement volume for each type of component: 60-100, $45-75,30-50$

- Range of maximum procurement volume for each component type: 100-180, 80-150, 60-100

- Range of component price for each component type (per unit): 35-52, 40-62, 49-72

- $\quad$ Range of transportation cost for each component type (per unit): 3-7, 3-7, 3-7

- Range of disruption probability of candidate suppliers: $18 \%-27 \%$.

The number of suppliers that could be selected with the constraints on the amount of demand of each component as well as the minimum and maximum procurement volume of each component set in this experiment must be at least 3. Moreover, owing to the constraint on the number of suppliers in formula (11), the number of suppliers that could be selected at one time is 3 or 4 .

As shown in Figure 6, based on the algorithm discussed in Section 5, there is confirmed difference in total procurement costs between the case of three suppliers and the case of four suppliers. With this experimental environment, the optimal number of suppliers is 3 .

The search accuracy of the proposed algorithm is also verified. Figure 7 shows the relationship between search times and total procurement cost with the proposed algorithm and a random improvement search, which randomly regenerates supplier combination and component allocation without evaluating the total procurement cost. In this experiment, the number of suppliers is set to 3, and the number of search times is set to 50.

The performance of the proposed algorithm is confirmed in Figure 7. It is found that the proposed algorithm requires only 17 search times, much less search times than random search, to reach a more accurate solution.

In addition, improvement effectiveness for different initial solutions as well as search speed of the proposed algorithm is also verified. Improvement solutions were searched with the proposed algorithm from five different random initial solutions by search time of 
100, 200, 500, 1,000, and 2,000. As shown in Figure 8, results of the initial and best solutions are plotted, respectively. Although the initial solutions are quite different, there is no clear difference among the best solutions by each search time. It is confirmed that the total procurement costs of all the best solutions are greatly improved from all 25 initial solutions. Furthermore, best solutions obtained by 100 search times and 2,000 search times are almost the same. So, search speed of the proposed algorithm is also confirmed since it was able to obtain a good solution with fewer search times.

Figure 6 Total procurement costs of the cases of three suppliers and four suppliers (see online version for colours)

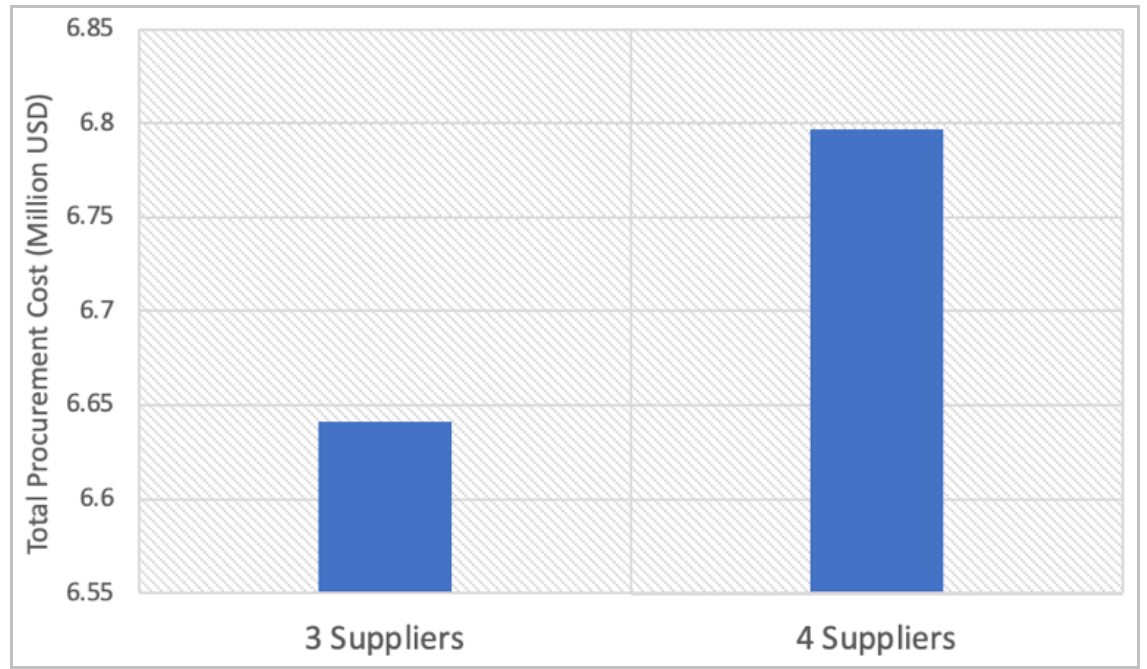

Figure 7 Total procurement cost with proposed algorithm and random search (see online version for colours)

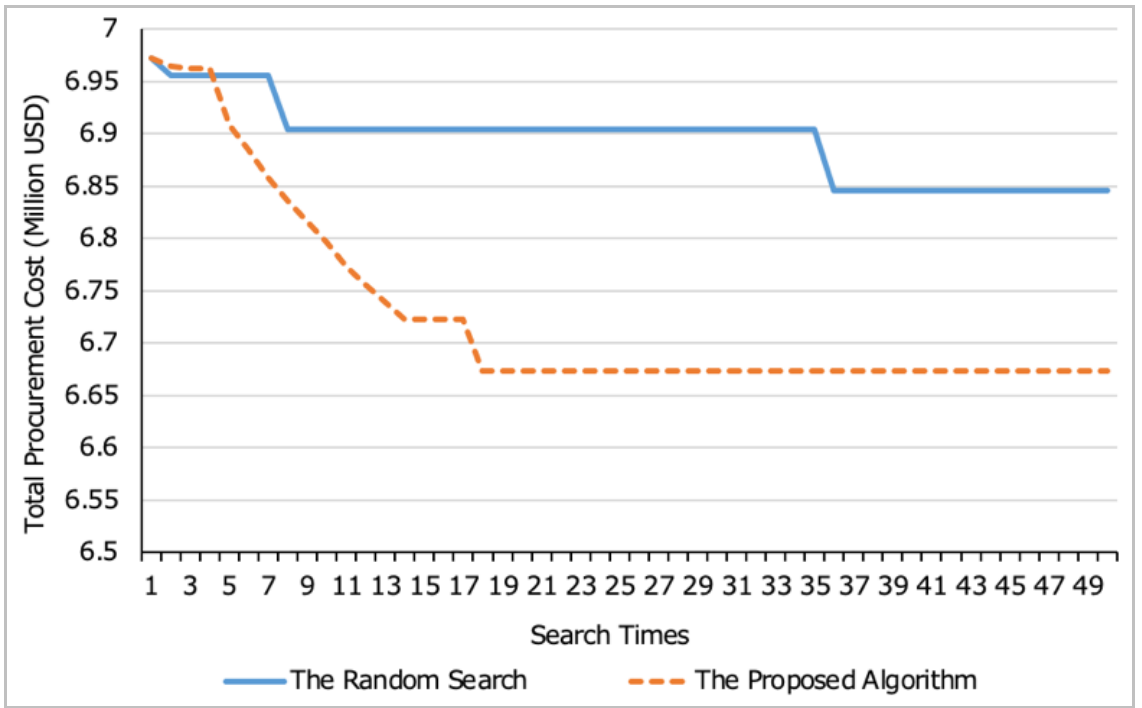


Figure 8 Improvement effect by different search times (see online version for colours)

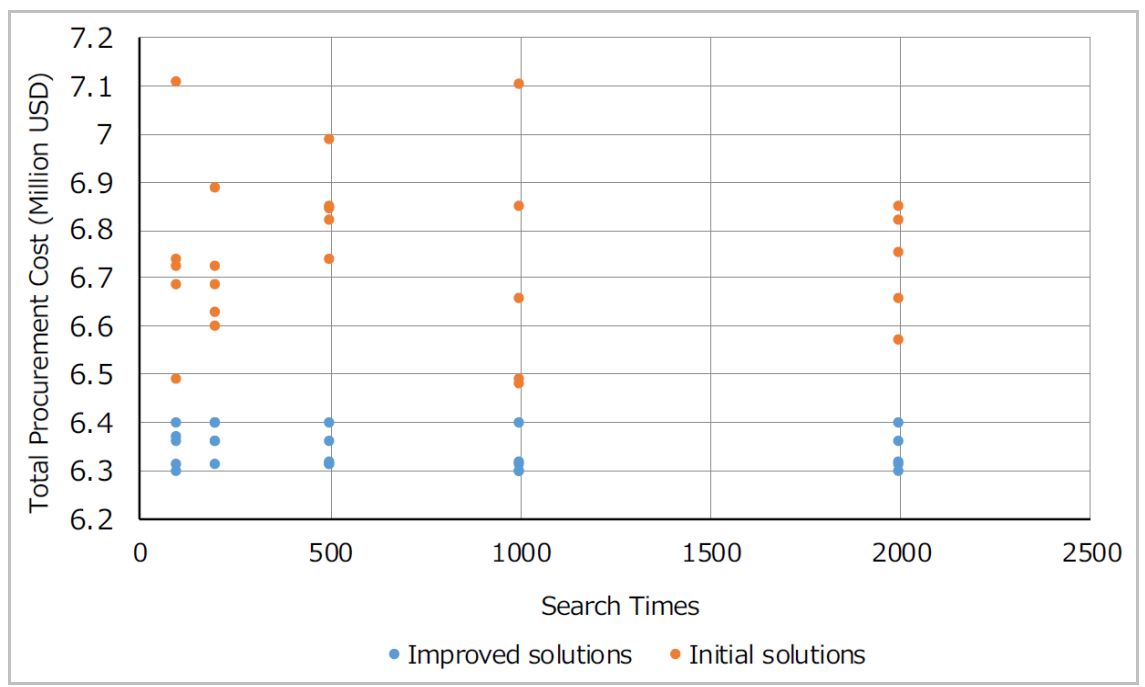

\section{Effectiveness verification of the sourcing decision with CR}

\subsection{Summary}

The disruption probability of all candidate suppliers is the largest factor in decisionmaking on countermeasures to supply disruptions. For a manufacturer, the higher the disruption probability of the supplier is, the higher is the opportunity loss and cost of countermeasures. In addition, the relationship between supply and demand in the component market is also considered a large impact factor. If the total supply is significantly more than the total demand, the manufacturer will have an advantage in determining the contract terms with suppliers. However, if the total supply is significantly less than the total demand, it will be difficult for the manufacturer to obtain good contract terms. In this study, the disruption probability of candidate suppliers and the supply demand relationship of the component market are considered to verify the effectiveness of CR. CR unit cost is considered as one of the contract terms.

In addition to the relationship between the location of supplier and component price discussed in 5.3, there is also a relationship between component price and production scale. Generally, the lower the component price, the larger is the supplier's production scale. Therefore, it could be considered that the reservation fee of production capacity of the supplier should be a little lower among the candidate suppliers. In addition to the experimental data introduced in 5.3, additional data for this verification are shown as follows.

\section{Detail of experiment data}

- $\quad$ Range of CR fee for each component type (per unit): 12-19.5, 13.5.22, and 15.5.25.5 
- $\quad$ Range of capacity utilisation cost for each component type (per unit): 42-78, 48-90, 57.6108

- $\quad$ Range of disruption probability of candidate suppliers: Low $(0 \%-17 \%)$, medium (18\%-27\%), high (28\%-47\%), extreme $(47 \%-100 \%)$.

Disruption probabilities of eight candidate suppliers are created randomly in each range (low, medium, high, extreme). Moreover, in a buyer's market (demand $<$ supply), the price for each component type is considered as half of that in a supplier's market (demand > supply). Therefore, the range of CR fee of each type of component (per unit) in a buyer's market would be 17.526 203124.536 .

A total of eight situations, including four disruption probability and two market features, are discussed in the experiment results. The unit of all prices in the experiment data is set as hundred USD.

\subsection{Results}

Total loss of manufacturer is considered as the evaluation index for the comparison between without CR model and the proposed with CR model. Opportunity loss, which has been discussed in Section 3, is considered as the loss in the without CR model. CR fee and the difference between capacity utilisation cost and component price of the disrupted supplier are considered as the loss in with the CR model.

Figure 9 shows the ratio of total loss for with CR model and without CR model in the buyer's market (demand < supply) and supplier's market (demand $>$ supply) under different disruption probabilities. The total loss is greater in with CR model than in without CR model under the low to medium disruption probability environment. Under a lower supply disruption probability environment, the reserved production capacity is not being utilised, even though the $\mathrm{CR}$ fee has been paid. As the disruption probability increases, the opportunity loss in without CR model increases rapidly. By contrast, opportunity loss can be mitigated due to the reserved production capacity with CR. In addition, in a supplier's market, manufacturers incur high costs in doing CR, since suppliers intend to sell their components instead of accepting CR because of the high market price of components. Furthermore, as previously discussed, in a lower disruption probability environment, the probability that the reserved capacity will not be utilised is high. Therefore, in the low disruption probability environment, the ratio of loss in the supplier's market is much higher than that in the buyer's market.

Figure 10 shows the average increase rate of total loss for with the CR model and without CR model in the buyer's market and the supplier's market with disruption probability increasing from low to extreme. In both market environments, the mitigation effect on loss increases in with CR model is confirmed. Compared with the environment of buyer's market, in the supplier's market, the increase rate of loss is quite higher with the probability of disruption increasing; nevertheless, the mitigation effect on loss increasing in with CR model is significant.

The CR fee could be considered as an incentive for suppliers to maintain their capacity for the specific manufacturers. It is a key factor in the CR model and may have a huge impact on the result of the sourcing decision. To clarify this, a simulation is conducted with a CR fee in three stages: low, medium, and high. The low stage is the half value of medium, and the high stage is the double value of the medium. 
Figure 9 Ratio of total loss in two markets under different disruption probabilities (see online version for colours)

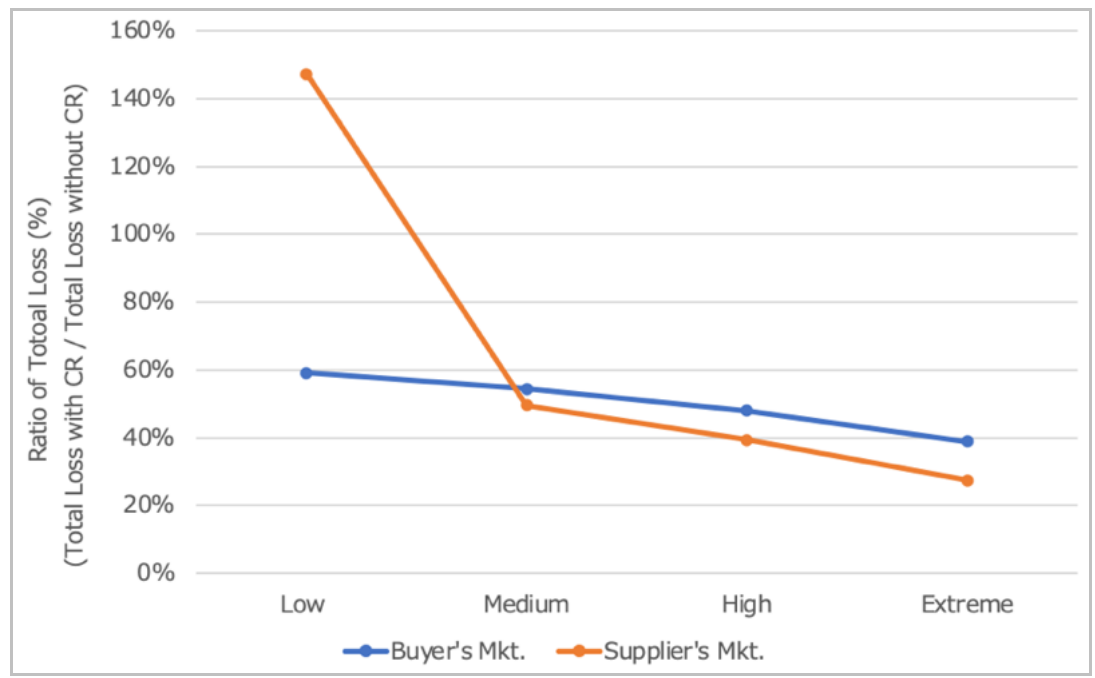

Figure 10 Average increase rate of total loss with increase in disruption probability (see online version for colours)

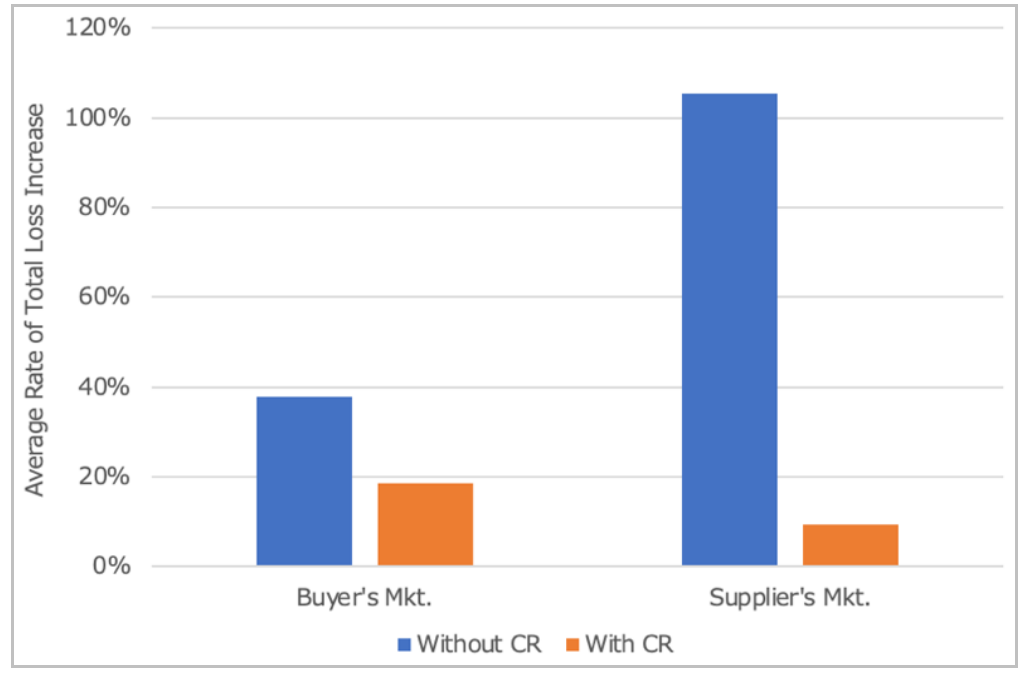

Figure 11 shows the result. The higher the CR fee is, the lower is the procurement cost with four suppliers rather than three suppliers. Compared with three suppliers, the required production capacity of each supplier for reservation would be less with four suppliers. Thus, the higher the CR unit fee is, the faster is the increase in the CR fee with three suppliers, compared to four suppliers. As a result, the increase in total procurement costs with three suppliers is rather obvious. 
Figure 11 Total procurement cost with different CR fee (see online version for colours)

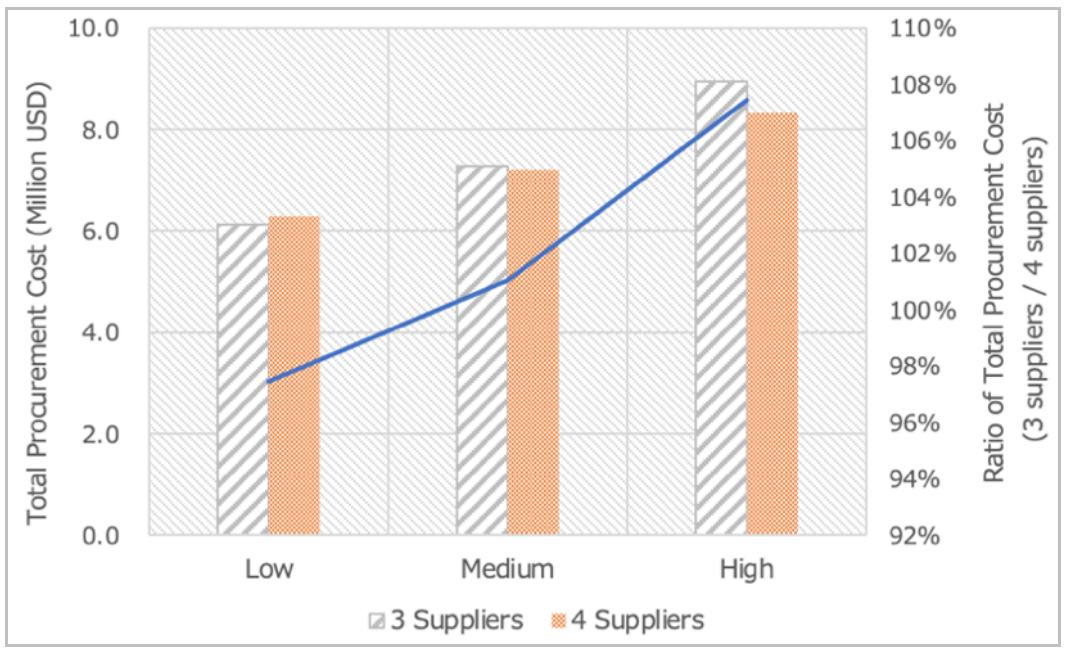

\section{Conclusions}

With the increase in supply chain disruption cases, manufacturers suffer from significant losses due to component procurement problems. In this study, as a countermeasure against supply chain disruptions for manufacturers, sourcing decision with supply CR was proposed. Once a supply disruption occurs, the unprocurable volume of components can be supplied by the undisrupted suppliers immediately with the production capacity, which has been reserved beforehand. Moreover, we discussed CR contracts with one supplier-one buyer structure and one supplier-multiple buyer structure in many previous studies. In this study, it was verified that the CR model could also be effective in multiple sourcing procurement situations. The effectiveness verification of the proposed CR model under component demand uncertainty as well as the consideration of restoration periods depending on different disasters will be our future work.

Production has been forcefully discontinued in a wide range of areas worldwide due to the recent COVID-19 pandemic. Because it is a global problem, it might be difficult to realise the loss mitigation for manufacturers with the proposed CR model in this study. Global supply chain network re-design and key suppliers re-positioning, considering the features of disasters, including the impact and restoration period, is necessary to be discussed with high priority in dealing with such a once-in-a-century crisis. 


\section{References}

Abginehchi, S. and Farahani, R.Z. (2010) 'Modelling and analysis for determining optimal suppliers under stochastic lead times', Applied Mathematical Modelling, Vol. 34, No. 5, pp.1311-1328.

Amir, A., Mohammad, M.P. and Abdul, S.S. (2018) 'An integrated fuzzy MOORA method and FMEA technique for sustainable supplier selection considering quantity discounts and supplier's risk', Journal of Cleaner Production, Vol. 190, pp.577-591.

Cheaitou, A. and Cheaytou, R. (2019) 'A two-stage capacity reservation supply contract with risky supplier and forecast updating', International Journal of Production Economics, Vol. 209, No. C, pp.42-60.

Chopra, S., Reinhardt, G. and Mohan, U. (2007) 'The importance of decoupling recurrent and disruption risks in a supply chain', Naval Research Logistics, Vol. 54, No. 5, pp.544-555.

Dadaet, M., Petruzzi, N.C. and Schwarz, L.B. (2007) 'A newsvendor's procurement problem when suppliers are unreliable', Manufacturing \& Service Operations Management, Vol. 13, No. 2, pp.227-243.

Hamdi, F., Dupont, L., Ghorbel, A. and Masmoudi, F. (2016) 'Supplier selection and order allocation under disruption risk', IFAC-Papers Online, Vol. 49, No. 12, pp.449-454.

Ho, W., Zheng, T., Yildiz, H. and Talluri, S. (2015) 'Supply chain risk management: a literature review', International Journal of Production Research, Vol. 53, No. 16, pp.5031-5069.

Hou, J., Zeng, A.Z. and Sun, L. (2017) 'Backup sourcing with capacity reservation under uncertain disruption risk and minimum order quantity', Computers and Industrial Engineering, Vol. 103, pp.216-226.

Jin, M. and Wu, S.D. (2007) 'Capacity reservation contracts for high-tech industry', European Journal of Operational Research, Vol. 176, No. 3, pp.1659-1677.

Masuda, T. and Mizuno, H. (2017) 'Study for risk management of disruption in supply chains', Proceedings of the School of Information and Telecommunication Engineering Tokai University, Vol. 10, No. 1, pp.25-35.

Meena, P.L. and Sarmah, S.P. (2016) 'Supplier selection and demand allocation under supply disruption risks', International Journal of Advanced Manufacturing Technology, Vol. 83, No. 1, pp.265-274.

Meena, P.L., Sarmah, S.P. and Sarkar, A. (2011) 'Sourcing decisions under risks of catastrophic event disruptions', Transportation Research Part E: Logistics and Transportation Review, Vol. 47, No. 6, pp.1058-1074.

Moghaddam, K.S. (2015) 'Fuzzy multi-objective model for supplier selection and order allocation in reverse logistics systems under supply and demand uncertainty', Expert Systems with Applications, Vol. 42, Nos. 15-16, pp.6237-6254.

Ross, D.F. (2015) Distribution Planning and Control, 3rd ed., Springer, New York.

Schmitt, A. (2011) 'Strategies for customer service level protection under multi-echelon supply chain disruption risk', Transportation Research Part B: Methodological, Vol. 45, No. 8, pp.1266-1283.

Schmitt, A.J., Snyder, L.V. and Schen, Z.J.M. (2010) 'Inventory systems with stochastic demand and supply: properties and approximations', European Journal of Operational Research, Vol. 206, No. 2, pp.313-328.

Takemoto, Y. and Arizono, I. (2019) 'Moral hazard problem and collaborative coordination in supply chain with capacity reservation contract', International Journal of Production Research, Vol. 58, No. 8, pp.2510-2526.

Tomlin, B. (2006) 'On the value of mitigation and contingency strategies for managing supply chain disruption risks', Management Science, Vol. 52, No. 5, pp.639-657.

Torabi, S.A., Baghersad, M. and Mansouri, S.A. (2015) 'Resilient supplier selection and order allocation under operational and disruption risks', Transportation Research Part E: Logistics and Transportation Review, Vol. 79, pp.22-48. 